\title{
Oral Lichenoid Contact Lesions to Mercury and Dental Amalgam-A Review
}

\author{
Helen McParland ${ }^{1}$ and Saman Warnakulasuriya ${ }^{2}$ \\ ${ }^{1}$ Oral Medicine Unit, Guy's and St Thomas' NHS Foundation Trust, London SE1 9RT, UK \\ ${ }^{2}$ Oral Medicine Unit, Department of Clinical \& Diagnostic Sciences, King's College London Dental Institute and \\ WHO Collaborating Centre for Oral Cancer, Bessemer Road, London SE5 9RS, UK
}

Correspondence should be addressed to Saman Warnakulasuriya, s.warne@kcl.ac.uk

Received 3 February 2012; Accepted 19 March 2012

Academic Editor: Jose G. Dorea

Copyright ( $) 2012$ H. McParland and S. Warnakulasuriya. This is an open access article distributed under the Creative Commons Attribution License, which permits unrestricted use, distribution, and reproduction in any medium, provided the original work is properly cited.

Human oral mucosa is subjected to many noxious stimuli. One of these substances, in those who have restorations, is dental amalgam which contains mercury. This paper focuses on the local toxic effects of amalgam and mercury from dental restorations. Components of amalgam may, in rare instances, cause local side effects or allergic reactions referred to as oral lichenoid lesions (OLLs). OLLs to amalgams are recognised as hypersensitivity reactions to low-level mercury exposure. The use of patch testing to identify those susceptible from OLL is explored, and recommendations for removing amalgam fillings, when indicated are outlined. We conclude that evidence does not show that exposure to mercury from amalgam restorations poses a serious health risk in humans, except for an exceedingly small number of hypersensitivity reactions that are discussed.

\section{Introduction}

Human oral mucosa is often subjected to many noxious stimuli, either hot or cold, acidic or alkaline substances, spiced or not so spicy foods. Among substance misusers, the oral mucosa is also in constant contact with tobacco, alcohol, or other substances taken through the mouth. In people with restored teeth, one material that is present in significant amounts is dental amalgam. Mercury is the primary ingredient incorporated during making an amalgam filling. This paper focuses on the local toxic effects of amalgam and mercury from dental restorations with particular reference to oral lichenoid reactions (lesions) as a result of low-level mercury exposure.

\section{Dental Amalgam}

Dental amalgam is an alloy composed of a mixture of approximately equal parts of liquid mercury and a powder consisting of silver $(\sim 22-32 \%)$, tin $(\sim 14 \%)$, copper $(\sim 8 \%)$, and other trace metals, including zinc [1]. Elemental mercury has been used in clinical dentistry since 1830s when it began to be used in fillings. Dental amalgam now has been used for well over one hundred and eighty years and remains the most commonly placed filling material in the world. Around the 1970s, approximately 22 million amalgam restorations were placed each year in NHS treatment in England and Wales [2], though the numbers have declined recently. On average in a British adult about 7 amalgam restorations can be found. Amalgam use though is declining; the main reason is that dental caries rates among school children and young adults are dropping. Improved alternative filling materials are also now available. A recent US survey, however, showed that $48 \%$ of US dentists were using dental amalgam [3]. Few countries like Sweden, Denmark, and Germany have restrictions on dental amalgam use, and Norway has completely banned amalgam. In the rest of the globe amalgam remains popular because it is strong, durable, and relatively inexpensive. It is considered a safe material but despite this concerns over its use remain because of the continuous low-level release of mercury, a substance which is known to be toxic [4-8]. Summaries of some selected research reports concerning amalgam fillings can be found at http://www.yourhealthbase.com/amalgams.html. 
Mercury vapour is released during insertion, condensation, and carving of amalgam. The amount of mercury in the restoration can be reduced by $6-10 \%$ by good condensation. During later dental interventions such as polishing a restoration may also result in release of further mercury. However, for newer amalgams polishing to improve the surface is no longer required [9], but in older adults with corroded amalgams this may still be necessary. A similar rise in mercury vapour occurs during removal of amalgams but that can be minimised by the use of adequate water coolants and high speed suction [10].

During the functional life of a restoration, chewing during eating can release mercury as a vapour or as a salt dissolved in saliva $[11,12]$, and studies have shown that the amount released directly correlates to the amount of amalgams present and their total surface area [12]. The daily absorbed dose of mercury from amalgam for the average individual is low, $1.2 \mu \mathrm{g}$ by inhaled mercury and $1.5 \mu \mathrm{g}$ by ingested mercury [13]. This represents less than of the daily amount derived from the food and nondental sources and falls below the threshold allowable or safe intake of mercury which has recently been reduced to 0.1 microgram/day per kilogram of body weight [14], but note is made that this EPA recommendation is mainly based on oral exposure to methyl mercury [15]. There are concerns, but no current scientific evidence, that mercury emitted from amalgam fillings may cause or worsen degenerative diseases such as amyotrophic lateral sclerosis (ALS), Alzheimer's disease, multiple sclerosis, and Parkinson's disease. Studies carried out regarding elemental mercury and cancer in humans are inconclusive due to lack of valid exposure data and confounding factors. In summary, the current scientific evidence does not show that exposure to mercury from amalgam restorations poses a serious health risk in humans, except for an exceedingly small number of hypersensitivity reactions [16] that are discussed below.

\section{Reactions of Oral Mucosa to Mercury and Amalgam}

Holmstrup [17] describes 3 distinct reactions to amalgam fillings in susceptible patients: type IV sensitivity, toxic reactions, and a much rarer phenomenon, acute or generalized sensitivity, the management of which differs considerably. These will be discussed separately below.

\section{Delayed or Type IV Hypersensitivity Reactions}

The most common reaction to amalgam is the development of oral lichenoid reactions/lesions (OLRs/OLLs) involving mucosae in direct contact with amalgam restorations (Figure 1).

An OLL generally represents a type IV hypersensitivity reaction $[18,19]$. Type IV hypersensitivity is often called a delayed type of hypersensitivity as the reaction takes a long period to develop and, in this case, could be months to years. Unlike the other types, it is not antibody mediated

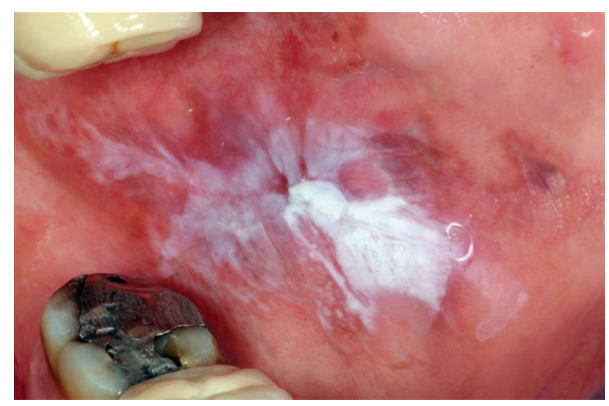

Figure 1: An oral lichenoid lesion or a toxic reaction on buccal mucosa found close to a large amalgam restoration. A positive patch test result to dental materials may confirm this to be an OLL.

but rather is a type of cell-mediated response. Mercury salts that accumulate in healthy and damaged oral mucosa [20] will cause this hypersensitivity reaction in only a susceptible minority of the population with resulting reticular white patches, papules, plaques, erosions, or ulceration, similar to that found in oral lichen planus (OLP)-hence the terminology lichenoid. These lesions can be asymptomatic or sore especially with hot or spicy food. Nonspecific toxic reactions, not as a result of hypersensitivity, can also manifest as OLL.

Hypersensitivity to dental amalgam is rare and according to Holmstrup is due to corrosion products of amalgam restorations, and it seems to be related to mercury in almost all cases, with only a few cases implicating silver, copper, or tin $[21,22]$.

OLLs of the oral mucosa caused by dental amalgam represent contact allergy. Contact allergies, although a common entity in skin, are relatively rare in oral mucosa [23]. Prolonged intimate contact of the oral mucosa with amalgam fillings over a long period, often many years, appears to be necessary, and in one study the mean age of patients with OLL was 54.6 years [24]. In order for a contact allergic reaction to be established, mercury salts and other metal ions which are leached from amalgam have to penetrate the epithelial lining and bind with host keratinocyte surface proteins. In susceptible individuals this results in a cellmediated response directed at basal keratinocytes [25-28]. Previous sensitisation of the individual must have occurred. In the case of mercury, dental amalgam may provide the first exposure but it may be also through other sources of mercury including disinfectants, cosmetics, dyes, foods, and vacuum preservatives [29]. It is possible that this is genetically determined by the HLA type but little evidence exists at present.

The pathophysiology of type $1 \mathrm{~V}$ hypersensitivity is complex. CD8+ cytotoxic T cells and CD4+ helper T cells recognize the antigen (metal elements in this case) in a complex with either type 1 or 2 major histocompatibility complex. The antigen-presenting cells normally are macrophages that secrete interleukins which stimulate the proliferation of further CD4+ T cells. These activated cells further induce the release of other type 1 cytokines, thus mediating the immune response. 


\section{Toxic Reactions}

Little is known about toxic reactions to irritants such as dental amalgam or its constituents but it is thought they can develop if an irritant substance is in direct contact with the mucosa over several years. Clinically they resemble OLLs (see Figure 1) which are caused by hypersensitivity reactions and can only be differentiated by exclusion based on a negative patch test [30]. Toxic reactions may be more common in amalgams with a higher zinc content [31].

\section{Clinical Features and Differential Diagnosis}

The lesions of OLL resemble those of oral lichen planus (OLP), and it is therefore necessary to exclude likely OLLs when making a diagnosis of OLP. While some authors do not differentiate the two $[32,33]$, we believe, along with others [24, 34-37], that the two conditions are distinct. OLP is a more widespread condition involving many anatomical sites within the oral cavity (or elsewhere including skin and genitalia) and distinct from OLL. Both OLP and OLL can be considered potentially malignant $[38,39]$. It is important for subsequent management to be able to accurately diagnose each condition.

Typically the clinical presentation in both conditions can be reticular white patches, papules, or plaques with or without erosions or ulcerated areas. The clinical diagnosis is further complicated because similar oral lesions can occur as a result of drug-related lichenoid reactions or as graft versus host disease (GVHD), discoid lupus erythematosus (DLE), and systemic lupus erythematosus (SLE). These conditions too have a similar clinical appearance. Diagnosis is facilitated by detailed history, clinical findings, and immunohistological findings. It is beyond this paper to discuss these other conditions.

OLLs caused by hypersensitivity to amalgam or its constituents typically have a clear anatomical relationship to the dental amalgam fillings [24], so they are usually unilateral and not symmetrical. They are most commonly seen on the buccal mucosae and tongue where the covering lining mucosa comes in contact with restorations. The gingivae, palate, or floor of mouth, being sites further away from restorations, are rarely affected, and patients almost never have associated cutaneous symptoms. These clinical features help to distinguish OLL from OLP and other conditions, but it can still be difficult for the clinician to make a clear distinction, if amalgam restorations are widespread in the mouth.

\section{Histopathology}

It is common practice to biopsy red and white lesions of the mouth to aid diagnosis [40] and to exclude dysplasia [41]. Classical histological features of an OLL are shown in Figure 2.

In 1973 Pinkus [42] coined the term "lichenoid tissue reaction" to describe the histological pattern featuring damage to keratinocytes, now referred to as apoptosis, infiltrate of

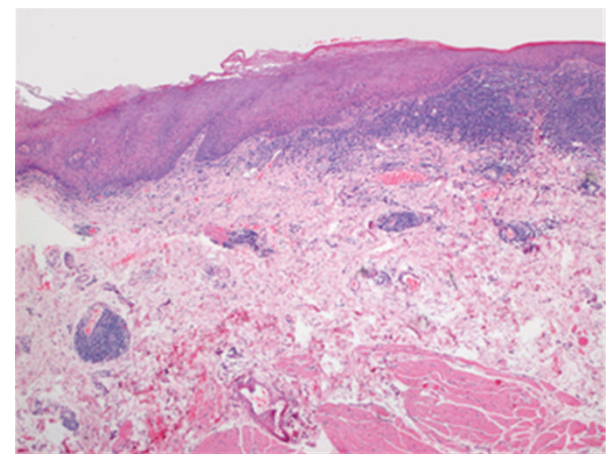

(a)

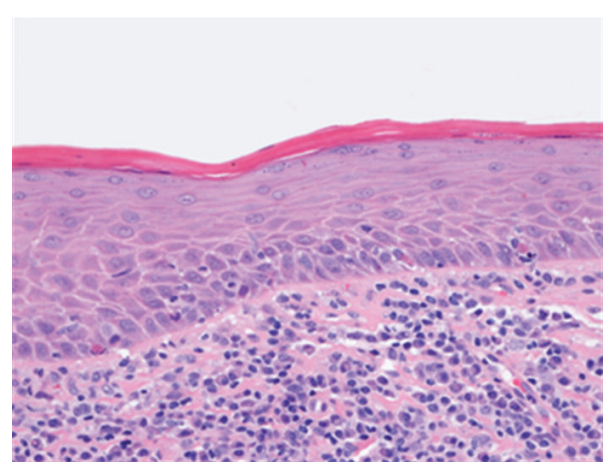

(b)

Figure 2: A biopsy taken from a lichenoid lesion. Photomicrographs show oral mucosa covered by stratified squamous epithelium which is atrophic and orthokeratinized. (a) The superficial lamina propria contains a dense and a well-demarcated lymphoplasmacytic infiltrate. A perivascular lymphoplasmacytic infiltrate also present in the deeper lamina propria. (b) There is thickening of basement membrane region, loss of well-defined basal cell layer, and frequent keratinocyte apoptosis. Photomicrographs; courtesy of Prof Edward Odell.

inflammatory cells in the connective tissue which may extend into the epithelium and keratosis or hyperkeratosis.

He described this histopathological pattern common to several diseases referred to above rather than a clinical entity. Since then, further histological features have been identified by Schiodt to distinguish, for example, oral DLE from OLP [43], namely, keratin plugging, atrophy of the rete processes, a deep inflammatory infiltrate, oedema in the lamina propria, and a thick PAS deposit in the basement membrane zone. It has also been suggested that a mixed cell subepithelial infiltrate and a deeper diffuse distribution in lamina propria can help to distinguish a lichenoid lesion [44].

Distinguishing between OLP and OLL remains a challenge. A study carried out by Thornhill et al. [24] confirmed the difficulty of making the distinction between OLL and OLP on purely histological grounds. Overall the pathologists were able to distinguish between the two conditions only a third of the time. Most pathologists report it is either OLP or OLL. 


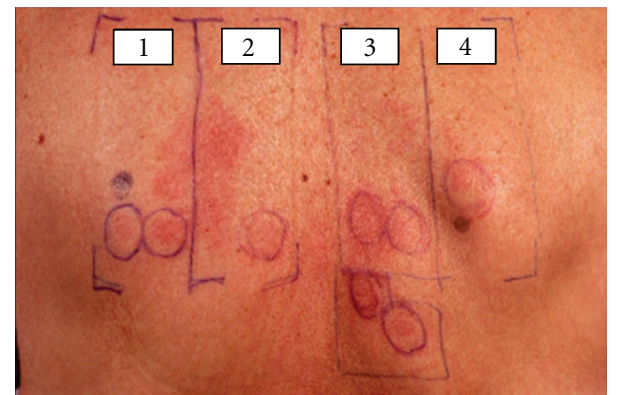

Figure 3: Positive skin reactions in the case illustrated include Row 1: cobalt chloride and balsam of peru, Row 2: nickel sulphate, Row 3: menthol, methyl methacrylate, palladium chloride, amalgam, and Row 4: ammoniated mercury

\section{Patch Testing}

Patch testing may be useful to identify those patients with suspected hypersensitivity reactions to amalgam or mercury $[24,45,46]$. However, studies investigating their usefulness have shown conflicting results [24]. It is likely that these earlier studies may have failed to clinically distinguish OLP from OLL when ordering patch testing. The test should be carried out in a specialist dermatology or oral medicine centre and is achieved by using commercially available kits which are typically placed on the skin of the back or fore arm in wells and held in place for 48 hours with hypoallergenic adhesive tape. The standard tests take into account that mercury from amalgam restorations may be in the form of metal, organic substances, or organic salts. Few patients react to all three forms. There is no worldwide consensus regarding the allergens used but generally it is accepted that $5 \%$ amalgam and $1 \%$ ammoniated mercury are suitable for screening [45]. The tests are usually carried out with an European series and includes other dental materials to screen for additional allergens.

The test results are generally read at 48 and 72 hours but evidence has shown that late readings at 10-14 days can capture previously missed positive reactions [34, 47, 48].

A skin reaction with erythema (Figure 3) and effusion with possible papulovesicles (eczema reaction) is considered a positive reaction [46].

Skin testing is preferable to mucosal testing due to a higher sensitivity and specificity and due to the simpler procedure [17]. Furthermore, allergen concentration on mucosa needs to be 5-12 times higher than that to develop skin reactions [49], and toxic reactions may occur at these high concentrations [17].

The routine use of patch testing for all patients with lichen planus like lesions should be avoided as the test itself is time consuming and may provoke sensitisation in the patient [50]. Holmstrup [21] lists the basis of patients requiring allergy testing as

(1) the presence of oral mucosal lesions as lichen planus or mucositis resistant to treatment,
(2) clear anatomical relationship between oral mucosal lesions and the suspected restorative material,

(3) lack of symmetry of affected sites.

The anatomical relationship appears to be the most powerful predictor of an OLL, and studies have shown that $70 \%$ of patients who had strong physical relationship of their mucosal lesions to amalgam tested positive to amalgam or ammoniated mercury, contrasting with $3.9 \%$ with weakly associated lesions. Patch tests, however, will not be $100 \%$ reliable as false positives will arise. $3.2 \%$ of the general population appear to be sensitised to dental amalgam or mercury. False negatives will arise for the minority of toxic reactions noted to mercury.

\section{Resolution of OLL following Removal of Amalgam Fillings}

Although a positive patch test may facilitate diagnosis of OLL caused by a hypersensitivity reaction, this can only be proved if resolution occurs after the offending amalgam has been removed. The resolution or partial resolution of lichenoid lesions following removal of amalgam restorations is illustrated in Figure 4. In one study lesions with direct contact with amalgam responded better when the restoration was removed than those exceeding the contact zone [51]. Some benefit was noted in $97 \%$ of such patients regardless of the patch test result but complete healing was seen more often in patients who had a positive patch test [51]. Some authors found a good response to replacement of amalgams in patients with the patch test reactions to mercury salts $[20,34,36]$ while others did not [52-54].

Amalgam removal had strongest effect on tongue lesions [51]. In another study relief of symptoms as early as 2-3 days after amalgam removal was found but this could take up to 5 weeks [55] or longer [56].

\section{Recommendations for Replacement following Detection of an OLL}

(1) OLL should be suspected clinically when

(i) lesions of the buccal mucosa or tongue are unilateral or not symmetrical,

(ii) lesions are in intimate contact with amalgam fillings,

(iii) lesions fail to heal following treatment.

They are unlikely in combination with

(i) cutaneous lesions,

(ii) desquamative gingivitis.

(2) Patch testing may help with diagnosis of OLL and is useful to check for sensitivities to other dental materials if amalgam fillings are to be replaced. We recommend that

(i) patch testing should be carried out in a specialist centre, 


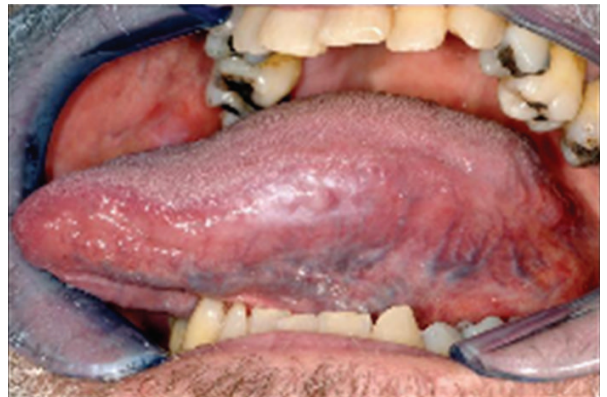

(a)

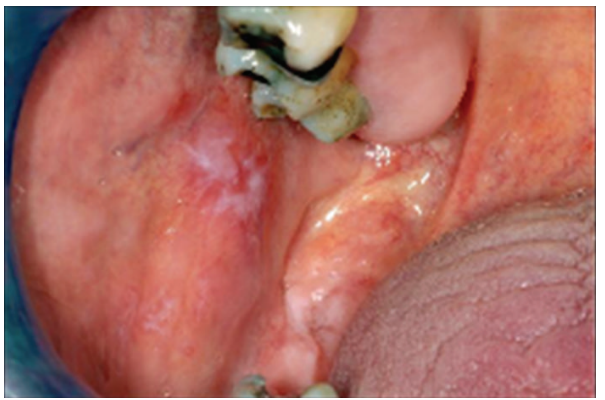

(a)

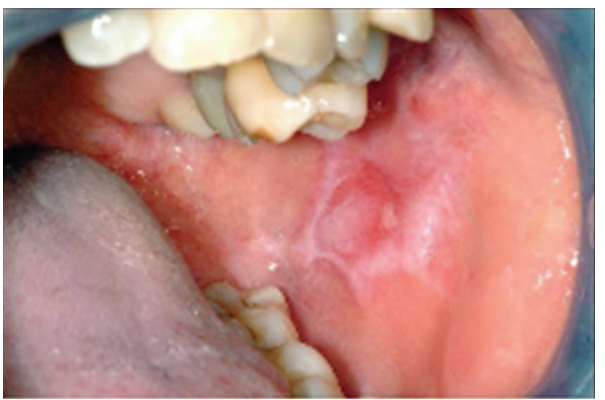

(a)

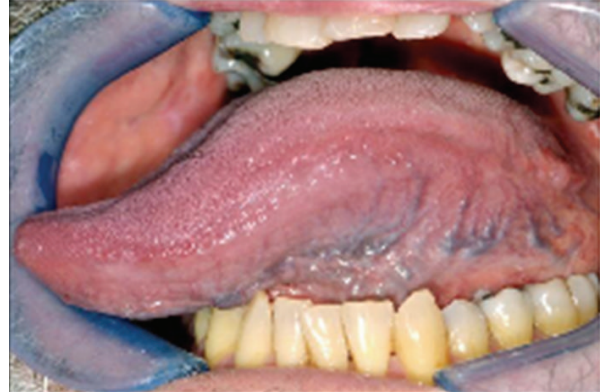

(b)

A

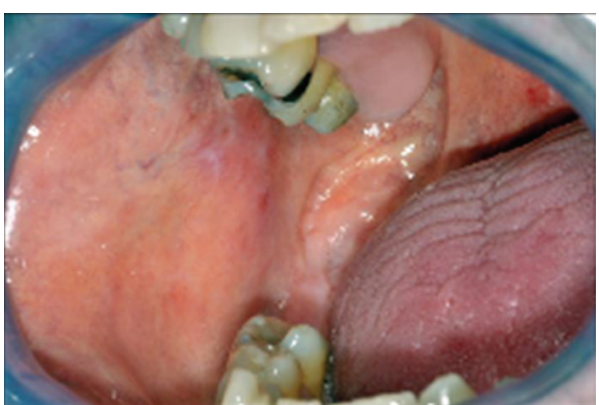

(b)

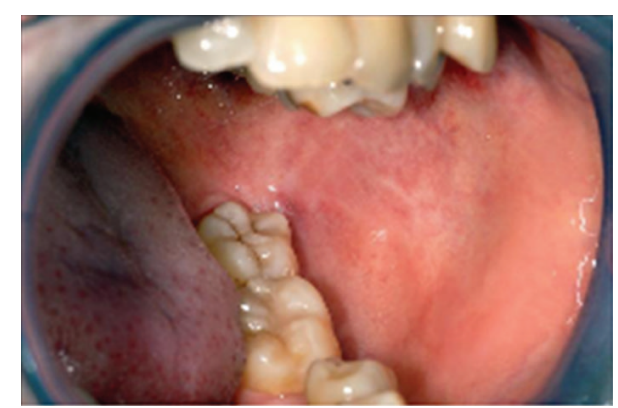

(b)

C

FIGURE 4: Illustrations showing resolution or partial resolution of lichenoid lesions following removal of amalgam restorations. All were in close contact with an amalgam filling. Panel A at the time of detection and Panel B after removal of the offending amalgam restoration.

(ii) products should be placed on the skin rather than mucosa,

(iii) 5\% amalgam, 1\% ammoniated mercury are suitable allergens,

(iv) test results are read late, 10-14 days in addition to usual 48 hours.

(3) Following a positive patch test, only amalgams in close contact to OLL should be removed. Placing a rubber dam during replacement is recommended.

(4) If the patch test is negative but there is a strong clinical suspicion of an OLL due to amalgam, either the crown can be covered to avoid amalgam contacting the mucosa or a single test amalgam can be removed to check healing before other amalgams are replaced.

\section{Acute or Generalized Sensitivity Reactions}

Reports on acute or generalized sensitivity reaction to amalgam or its constituents are rare. One report stated that this could occur in susceptible individuals after inhalation or absorption of mercury vapour, for example, during or directly after placement of an amalgam filling. Symptoms include the development of a cutaneous, erythematous, urticarial rash affecting the face and limbs, usually on the flexural aspect [17]. These reactions are on the same side of the body as the dental intervention. Acute mucosal reactions intraorally are much more rare but may present as vesicles which rupture to form erosions [57]. Symptoms usually appear within hours [57-62] of amalgam placement or removal and typically are self-limiting and resolve over the course of a few days [61]. 
The nature of these reactions is not fully understood but it is thought they may represent type 1 hypersensitivity reactions [63], and some authors recommend the use of antihistamine therapy for relief of symptoms $[60,64]$.

Following an acute reaction, unlike with OLL, existing amalgams can remain in place and need only be removed if symptoms persist [44] but alternatives to amalgam should be chosen for future restorations. If amalgam removal is necessitated, the use of rubber dam and high speed suction and copious water coolant is recommended and antihistamine therapy given prior to treatment $[56,60]$.

For acute reactions, patch testing for amalgam is not recommended as it is not useful in type I sensitivity.

\section{Conclusions}

Health policy for amalgams can be based on scientific reports from many advisory and regulatory bodies. Based on the guidance given by The Centre for Disease Control (May 2010) [16], at present, there is scant evidence that the health of the vast majority of people with amalgam is compromised nor that removing amalgam fillings has a beneficial effect on health. There is insufficient evidence of a link between dental mercury and health problems [65], except in rare instances of an allergic reaction. The WHO highlights the importance of reporting systems on adverse side effects of dental materials during dental care [66]. This paper highlights the diagnosis and clinical findings of contact allergic reactions to mercury and amalgam. This update should enable dentists to undertake appropriate measures when patients have a suspected or proven contact allergy on their oral mucosa to mercury or other metallic constituents of amalgam.

\section{Acknowledgments}

The authors thank Pam Chart and Barbara Burke for secretarial assistance.

\section{References}

[1] S. J. Jensen, "Maximum contents of mercury in dental silver amalgams," Scandinavian Journal of Dental Research, vol. 93, no. 1, pp. 84-88, 1985.

[2] Office of Health Economics, Compedium of Health Statistics, Office of Health Economics, London, UK, 7th edition, 1989.

[3] http://thewealthydentist.com/surveyresults/16_mercuryamalgam_results.htm.

[4] M. Hanson and J. Pleva, "The dental amalgam issue. A review," Experientia, vol. 47, no. 1, pp. 9-22, 1991.

[5] ADA Council on Scientific Affairs, "Dental amalgam update on safety concerns," The Journal of the American Dental Association, vol. 129, pp. 494-503, 1998.

[6] S. B. Corbin and W. G. Kohn, "The benefits and risks of dental amalgam: current findings reviewed," The Journal of the American Dental Association, vol. 125, no. 4, pp. 381-388, 1994.

[7] B. M. Eley, "The future of dental amalgam: a review of the literature Part 6: possible harmful effects of mercury from dental amalgam," British Dental Journal, vol. 182, no. 12, pp. 455459, 1997.
[8] M. Levy, "Dental amalgam: toxicological evaluation and health risk assessment," Journal of the Canadian Dental Association, vol. 61, no. 8, pp. 667-671, 1995.

[9] K. O. Frykholm, "On mercury from dental amalgam;its toxic and allergenic effects and some comments on occupational hygiene," Acta Odontologica Scandinavica, vol. 15, supplement 22, pp. 1-108, 1957.

[10] J. M. Richards and P. J. Warren, "Mercury vapour released during the removal of old amalgam restorations," British Dental Journal, vol. 159, no. 7, pp. 231-232, 1985.

[11] M. J. Vimy and F. L. Lorscheider, "Serial measurements of intra-oral air mercury: estimation of daily dose from dental amalgam," Journal of Dental Research, vol. 64, no. 8, pp. 10721075, 1985.

[12] A. Berglund, "Estimation by a 24-hour study of the daily dose of intra-oral mercury vapor inhaled after release from dental amalgam," Journal of Dental Research, vol. 69, no. 10, pp. 1646-1651, 1990.

[13] J. R. Mackert Jr., "Factors affecting estimation of dental amalgam mercury exposure from measurements of mercury vapor levels in intra-oral and expired air," Journal of Dental Research, vol. 66, no. 12, pp. 1775-1780, 1987.

[14] T. W. Clarkson, L. Magos, and G. J. Myers, "The toxicology of mercury-current exposures and clinical manifestations," New England Journal of Medicine, vol. 349, no. 18, pp. 17311737, 2003.

[15] Environmental Protection Agency, Reference Dose for Chronic Oral Exposure to Methylmercury, Integrated Information System, Greenbelt, Md, USA, 2001.

[16] http://www.cdc.gov/oralhealth/publications/factsheets/amalgam.htm.

[17] P. Holmstrup, "Reactions of the oral mucosa related to silver amalgam: a review," Journal of Oral Pathology and Medicine, vol. 20, no. 1, pp. 1-7, 1991.

[18] R. A. Cawson and E. W. Odell, Eds., Cawson's Essentials of Oral Medicine and Pathology, Churchill Livingston, London, UK, 2008.

[19] M. Jolly, A. J. Moule, R. W. Bryant, and S. Freeman, "Amalgam-related chronic ulceration of oral mucosa," British Dental Journal, vol. 160, no. 12, pp. 434-437, 1986.

[20] J. Bolewska, P. Holmstrup, B. Moller-Madsen, B. Kenrad, and G. Danscher, "Amalgam associated mercury accumulations in normal oral mucosa, oralmucosal lesions of lichen planus and contact lesions associated with amalgam," Journal of Oral Pathology and Medicine, vol. 19, no. 1, pp. 39-42, 1990.

[21] P. Holmstrup, "Oral mucosa and skin reactions related to amalgam," Advances in Dental Research, vol. 6, pp. 120-124, 1992.

[22] J. Banoczy, B. Roed Petersen, J. J. Pindborg, and J. Inovay, "Clinical and histologic studies on electrogalvanically induced oral white lesions," Oral Surgery Oral Medicine and Oral Pathology, vol. 48, no. 4, pp. 319-323, 1979.

[23] K. Merritt, International Workshop: Biocompatibility, Toxicity and Hypersensitivity to Alloy Systems Used in Dentistry, 1986.

[24] M. H. Thornhill, M. N. Pemberton, R. K. Simmons, and E. D. Theaker, "Amalgam-contact hypersensitivity lesions and oral lichen planus," Oral Surgery, Oral Medicine, Oral Pathology, Oral Radiology, and Endodontics, vol. 95, no. 3, pp. 291299, 2003.

[25] M. H. Thornhill, "Immune mechanisms in oral lichen planus," Acta Odontologica Scandinavica, vol. 59, no. 3, pp. 174-177, 2001. 
[26] L. R. Eversole, "Immunopathogenesis of oral lichen planus and recurrent aphthous stomatitis," Seminars in Cutaneous Medicine and Surgery, vol. 16, no. 4, pp. 284-294, 1997.

[27] S. R. Porter, A. Kirby, I. Olsen, and W. Barnett, "Immunological aspects of dermal and oral lichen planus; a review," Oral Surgery, Oral Medicine, Oral Pathology, Oral Radiology, and Endodontology, vol. 83, pp. 358-366, 1997.

[28] P. B. Sugerman, N. W. Savage, L. J. Walsh et al., "The pathogenesis of oral lichen planus," Critical Reviews in Oral Biology and Medicine, vol. 13, no. 4, pp. 350-365, 2002.

[29] S. Enestrom and P. Hultman, "Does amalgam affect the immune system? A controversial issue," International Archives of Allergy and Immunology, vol. 106, no. 3, pp. 180-203, 1995.

[30] M. Jontell and P. Holmstrup, "Red and white lesions of the oral mucosa," in Burket's Oral Medicine, M. S. Greenberg, M. Glick, and J. A. Ship, Eds., pp. 77-78, BC Decker, Hamilton, Canada, 2008.

[31] M. Kaga, N. S. Seale, T. Hanawa, J. L. Ferracane, and T. Okabe, "Cytotoxicity of amalgams," Journal of Dental Research, vol. 67, no. 9, pp. 1221-1224, 1988.

[32] I. M. C. Lundstrom, "Allergy and corrosion of dental materials in patients with oral lichen planus," International Journal of Oral Surgery, vol. 13, no. 1, pp. 16-24, 1984.

[33] J. A. Yiannias, R. A. El-Azhary, J. H. Hand, S. Y. Pakzad, and R. S. Rogers, "Relevant contact sensitivities in patients with the diagnosis of oral lichen planus," Journal of the American Academy of Dermatology, vol. 42, no. 2, pp. 177-182, 2000.

[34] J. Laine, K. Kalimo, and R. P. Happonen, "Contact allergy to dental restorative materials in patients with oral lichenoid lesions," Contact Dermatitis, vol. 36, no. 3, pp. 141-146, 1997.

[35] P. O. Ostman, G. Anneroth, and A. Skoglund, "Oral lichen planus lesions in contact with amalgam fillings: a clinical, histologic, and immunohistochemical study," Scandinavian Journal of Dental Research, vol. 102, no. 3, pp. 172-179, 1994.

[36] M. Pecegueiro, M. F. Sachse, J. Amaro, P. Farinha, and I. Fonseca, "Oral lichen planus versus oral lichenoid eruption as a manifestation of contact allergy," Contact Dermatitis, vol. 40, no. 6, pp. 333-334, 1999.

[37] J. Bratel, M. Hakeberg, and M. Jontell, "Effect of replacement of dental amalgam on oral lichenoid reactions," Journal of Dentistry, vol. 24, no. 1-2, pp. 41-45, 1996.

[38] M. D. Mignogna, L. Lo Muzio, L. Lo Russo, S. Fedele, E. Ruoppo, and E. Bucci, "Clinical guidelines in early detection of oral squamous cell carcinoma arising in oral lichen planus: a 5-year experience," Oral Oncology, vol. 37, no. 3, pp. 262$267,2001$.

[39] E. H. van der Meij, H. Mast, and I. van der Waal, "The possible premalignant character of oral lichen planus and oral lichenoid lesions: a prospective five-year follow-up study of 192 patients," Oral Oncology, vol. 43, no. 8, pp. 742-748, 2007.

[40] R. J. Melrose, "Failure to diagnose pathology: an avoidable complication in oral and maxillofacial surgery," Oral and Maxillofacial Surgery Clinics of North America, vol. 23, no. 3, pp. 465-473, 2011.

[41] S. Warnakulasuriya, J. Reibel, J. Bouquot, and E. Dabelsteen, "Oral epithelial dysplasia classification systems: predictive value, utility, weaknesses and scope for improvement," Journal of Oral Pathology and Medicine, vol. 37, no. 3, pp. 127-133, 2008.

[42] H. Pinkus, "Lichenoid tissue reactions; a speculative review of the clinical spectrum of epidermal cell damage with special reference to erythema dyschromium pestans," Archives of Dermatology, vol. 107, pp. 840-843, 1973.
[43] M. Schiodt, "Oral discoid lupus erythematosus. III. A histopathologic study of sixty-six patients," Oral Surgery Oral Medicine and Oral Pathology, vol. 57, no. 3, pp. 281-293, 1984.

[44] L. W. Solomom, T. N. Helm, C. Stevens, M. E. Neidas, and V. Kumar, "Clincal and immunopathiological findings in oral lichen planus pemphigoides," Oral Surgery, Oral Medicine, Oral Pathology, Oral Radiology \& Endodontics, vol. 103, pp. 808-813, 2007.

[45] S. Fregert, Manual of Contract Dermatitis, Copenhagen, Munksgaard, Danmark, 2nd edition, 1981.

[46] D. Ditrichova, S. Kapralova, and M. Tichy, "Oral lichenoid lesions and allergy to dental materials," Biomedical Papers of the Medical Faculty of the University Palacký, Olomouc Czechoslovakia, vol. 15, no. 2, pp. 333-339, 2007.

[47] P. Koch and F. A. Bahmer, "Oral lesions and symptoms related to metals used in dental restorations: a clinical, allergological, and histologic study," Journal of the American Academy of Dermatology, vol. 41, no. 3, pp. 422-430, 1999.

[48] H. Mobacken, K. Hersle, K. Sloberg, and H. Thilander, "Oral lichen planus: hypersensitivity to dental restoration material," Contact Dermatitis, vol. 10, no. 1, pp. 11-15, 1984.

[49] G. Lüders, "Exogen induzierte erkrarkungen der mundschleimhaut," Zeitschrift für Hautkrankheiten, vol. 62, pp. 603$612,1987$.

[50] W. G. Shafer, M. K. Hine, and B. M. Levy, A Textbook of Oral Pathology, Saunders, Philadelphia, Pa, USA, 4th edition, 1983.

[51] A. Dunsche, I. Kastel, H. Tenheyden, I.N.G. Springer, E. Christopers, and J. Brash, "Oral lichenoid reactions associated with amalgam; improvement after amalgam removal," British Journal of Dermatology, vol. 148, pp. 70-76, 2003.

[52] P. O. Ostman, G. Anneroth, and A. Skoglund, "Oral lichen planus lesions in contact with amalgam fillings: a clinical, histologic, and immunohistochemical study," Scandinavian Journal of Dental Research, vol. 102, no. 3, pp. 172-179, 1994.

[53] A. Skoglund, "Value of epicutaneous patch testing in patients with oral, mucosal lesions of lichenoid character," Scandinavian Journal of Dental Research, vol. 102, no. 4, pp. 216-222, 1994.

[54] K. Kalimo, "Oral lichenoid lesions caused by allergy to mercury in amalgam fillings," Contact Dermatitis, vol. 35, no. 1, p. 69, 1996.

[55] E. R. Smart, R. I. Macleod, and C. M. Lawrence, "Resolution of lichen planus following removal of amalgam restorations in patients with proven allergy to mercury salts: a pilot study," British Dental Journal, vol. 178, no. 3, pp. 108-112, 1995.

[56] S. H. Ibbotson, E. L. Speight, R. I. Macleod, E. R. Smart, and C. M. Lawrence, "The relevance and effect of amalgam replacement in subjects with oral lichenoid reactions," British Journal of Dermatology, vol. 134, no. 3, pp. 420-423, 1996.

[57] L. R. Eversole, "Allergic stomatitides," Journal of Oral Medicine, vol. 34, no. 4, pp. 93-102, 1979.

[58] W. A. Wiltshire, M. R. Ferreira, and A. J. Ligthelm, "Allergies to dental materials," Quintessence International, vol. 27, no. 8, pp. 513-520, 1996.

[59] J. Thomson and J. A. Russell, "Dermatitis due to mercury following amalgam dental restorations," British Journal of Dermatology, vol. 82, no. 3, pp. 292-297, 1970.

[60] F. A. Wright, "Allergic reaction to mercury after dental treatment," New Zealand Dental Journal, vol. 67, no. 310, pp. 251252, 1971.

[61] H. Nakayama, F. Niki, M. Shono, and S. Hada, "Mercury exanthem," Contact Dermatitis, vol. 9, no. 5, pp. 411-417, 1983. 
[62] A. J. Duxbury, R. D. Ead, S. McMurrough, and D. C. Watts, "Allergy to mercury in dental amalgam," British Dental Journal, vol. 152, no. 2, pp. 47-48, 1982.

[63] B. McGivern, M. Pemberton, E. D. Theaker, J. A. Buchanan, and M. H. Thornhill, "Delayed and immediate hypersensitivity reactions associated the use of amalgam," British Dental Journal, vol. 188, no. 2, pp. 73-76, 2000.

[64] R. R. White and R. L. Brandt, "Development of mercury hypersensitivity among dental students," The Journal of the American Dental Association, vol. 92, no. 6, pp. 1204-1207, 1976.

[65] J. Eyeson, I. House, Y. H. Yang, and K. A. A. S. Warnakulasuriya, "Relationship between mercury levels in blood and urine and complaints of chronic mercury toxicity from amalgam restorations," British Dental Journal, vol. 208, no. 4, p. E7, 2010.

[66] World Health Organization, "Future use of materials for dental restoration: report of the meeting convened at WHO HQ," Geneva, Switzerland, prepared by Dr. Poul Erik Petersen, 2009. 

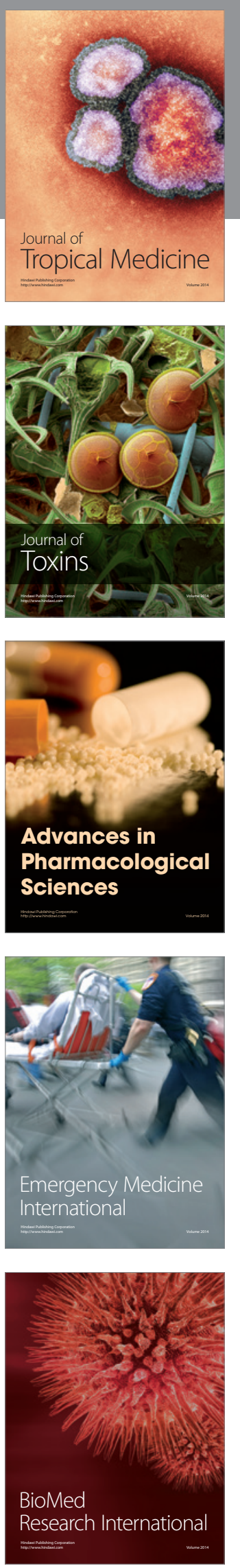
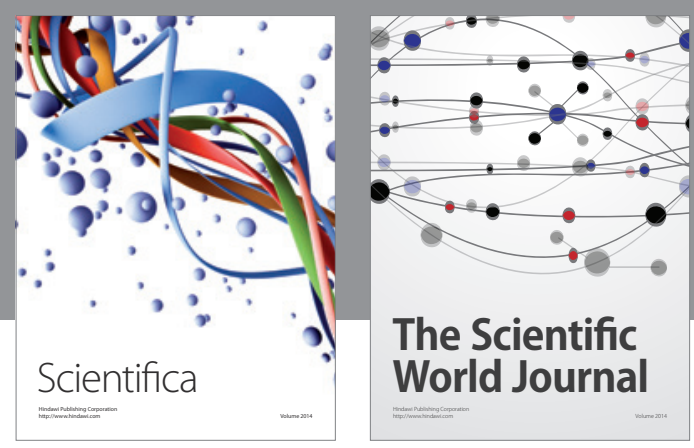

The Scientific World Journal
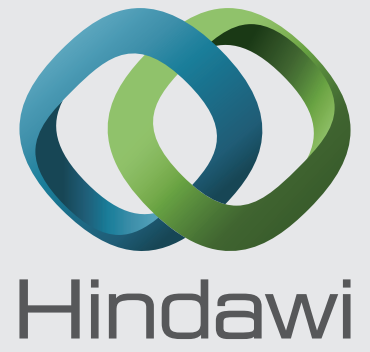

Submit your manuscripts at

http://www.hindawi.com
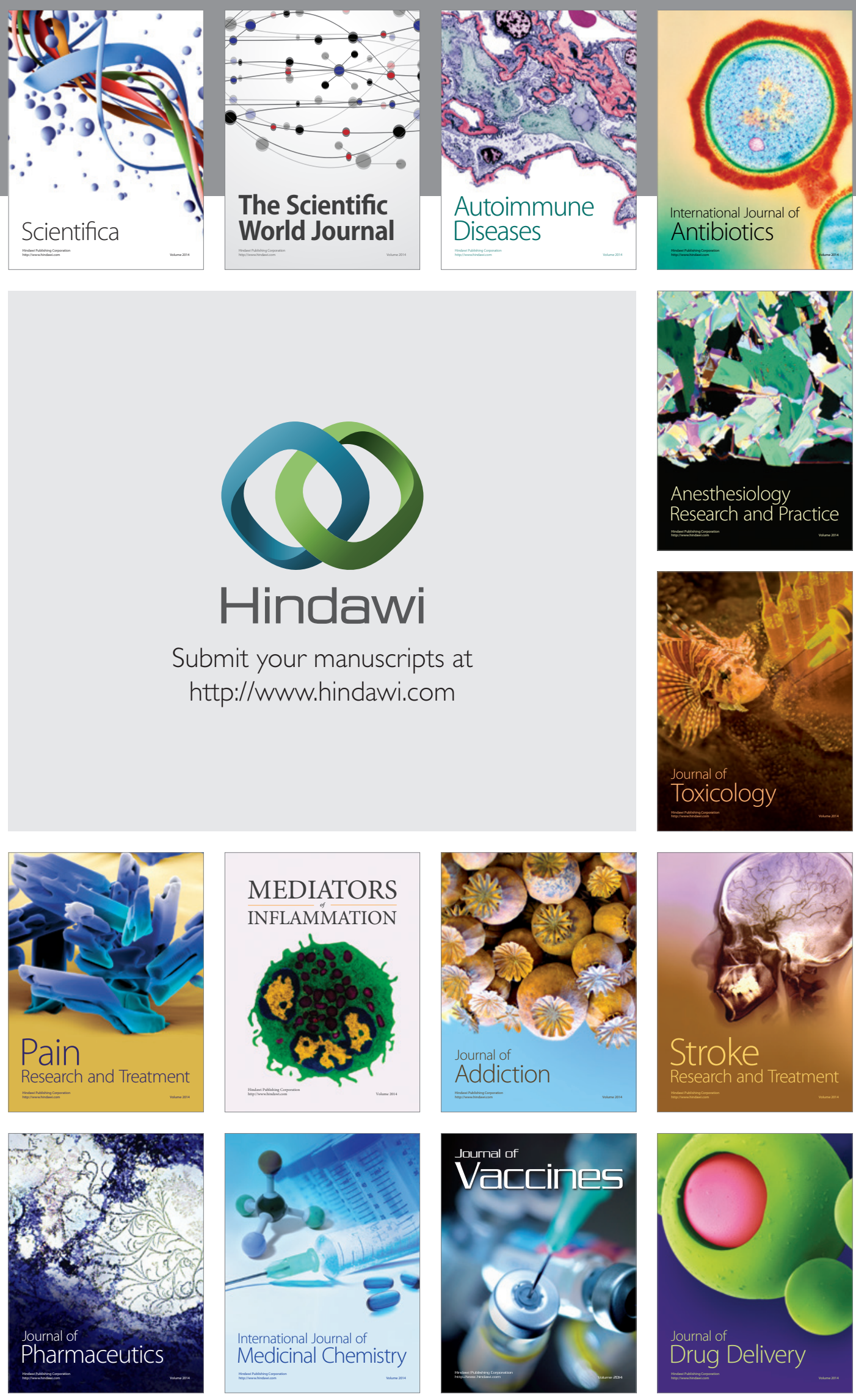